\title{
Location of Wrist Extension Movement in SMEG
}

\author{
Qi Wang
}

\author{
Lanzhou University of Technology, Lanzhou 730050, China \\ e-mail: qiqi691@lut.cn
}

Keywords: wrist extensor action, large Intestine channel of hand yangming, proportional bone measurement.

\begin{abstract}
The extraction of pure sMEG signals is a bottleneck problem of functional electrical limb rehabilitation. Comparing human active wrist extensor muscle group and large intestine channel of hand yangming, we designed the experiment to search pure wrist extensor action site by using their conjunction points. The result show that pure wrist extensor EMG can be found at shou san li(LI10)and shang lian(LI9) in the large intestine channel of hand yangming by proportional bone measurement. This method ensures the accuracy of the action site and not affected by human somatotype. The experiment is the foundation of stump rehabilitation research and has guiding significance for locating other action sites required by rehabilitation research.
\end{abstract}

\section{Introduction}

Stroke is an acute disease caused by blood vessels that supply blood to the brain. According to information released by WHO, stroke is one of the three major factors that cause sudden death in 40 countries in the world and occupies the first place in China. China Disabled Persons 'Federation(CDFP) survey data show that there are 7 million patients with hemiplegia caused by stroke in our country and about 4 million and 500 thousand survivors had a sequelae of limb paralysis. The patients who loss the motor function for paralysis bring heavy burden to the family and society. So, how to build the patients' motor function is an important topic to improve the quality of their life and to reduce the social pressure.

The plasticity of the central nervous system indicates the possibility of functional reconstruction after brain injury through regular rehabilitation training. Recent years, there have been many domestic and foreign applications of surface electromyography (sEMG) technology to research stroke patients with hemiplegia. By extracting the characteristic parameters of sEMG, literatures have proved that there are different degrees of correlation between the active state and functional state of muscle [1] and muscle contraction training can help patients with hemiplegia complete daily living activities [2].

Although appropriate rehabilitation training can relieve muscle spasm, make the limb movements tend to be coordinated. However, inappropriate muscle strength training may aggravate the spasm and leave the abnormal movement patterns, then hinder to improve the ability of patients' activities. Functional electrical stimulation(FES), which can repair lower limb function of paralysis patients, has become one of the important treatment methods of the residual limb rehabilitation [3,4]. So, our idea on stump motion reconstruction is using normal muscle movement patterns and motion sites corresponding to pure actions to adjust the corresponding muscle condition of the patient with hemiplegia. The selection of pure action sites which obtained by stimulating body surface is very important in the whole study. Wrist extension location is an example to introduce the research ideas.

\section{Method}

Theoretical support for site determination.Comparing of human arm anatomy diagram and hand Yangming large intestine meridian figure in figure1, the acupoints on hand Yangming large intestine meridian mainly distributed in wrist extension active muscle groups, and Quchi(LI 11), shou san li, shang lian are acupoints for acupuncture treatment of hemiplegia. It provides a 
theoretical basis for the detection of human wrist extensor action site in large intestine channel of hand yangming.

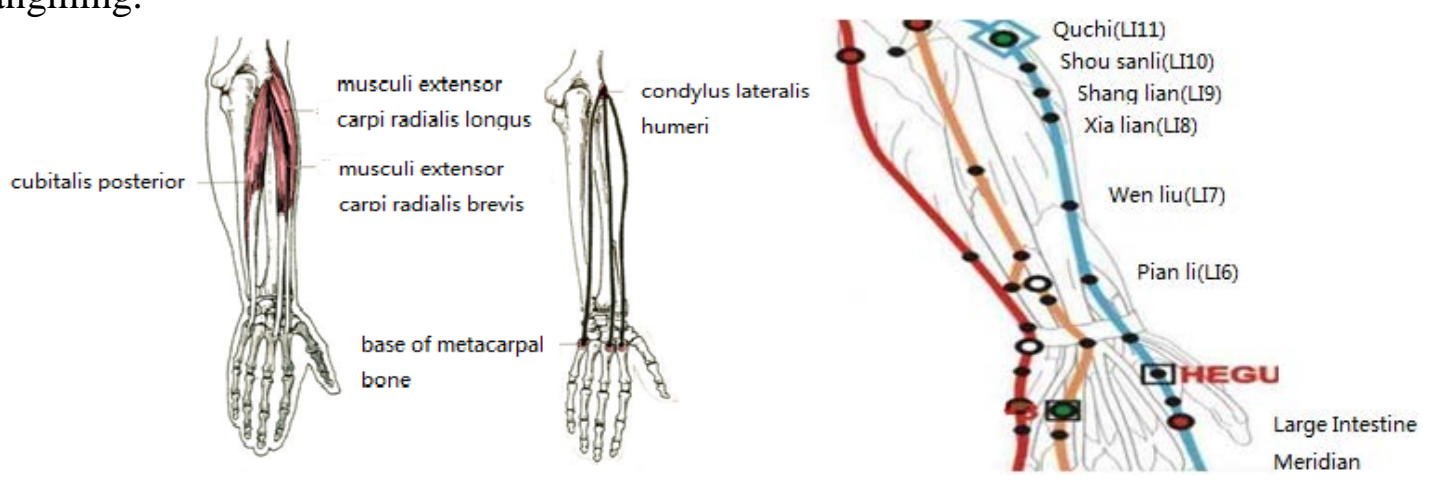

Fig 1 anatomical sketch of forearm and the distribution map of YangMing Meridian

Reliability analysis.Because of skin at acupoints with low resistivity, low current can produce high output density on the central nervous system and play a stimulating role. On the other hand, stimulating acupoints is a way to treat illness in Chinese medicine. Therefore, the implementation of electric acupoint stimulation has no security risks on the surface of the human body. The stimulus signal for the human body requires less tissue damage and charge balance. So, we adopted the asymmetric bidirectional stimulus signal which has equal positive and negative area as stimulus signal, shown in figure 2 .

proportional bone measurement [5] is a kind of acupuncture point positioning method in traditional Chinese medicine. To avoid the effect of body size on the EMG detection site, this method located acupoints by subjects' body inch. Thus, the consistency of the stimulus site selection is guaranteed. The location of the electrodes can be recorded by the Plus Image-Pro software., and the dimensions to be measured are shown in Fig 3. The stipulate in The people's Republic of China National Standard acupoints (2) said that from elbow stripes to carpometacarpal lateral stripes is 12 inch. So, the normalized and reference excitation electrode sites can be calculated by formula (1), and the measuring unit is inch.

electrode site $=($ excitation electrode to rasceta length $/$ Forearm length $) * 12$

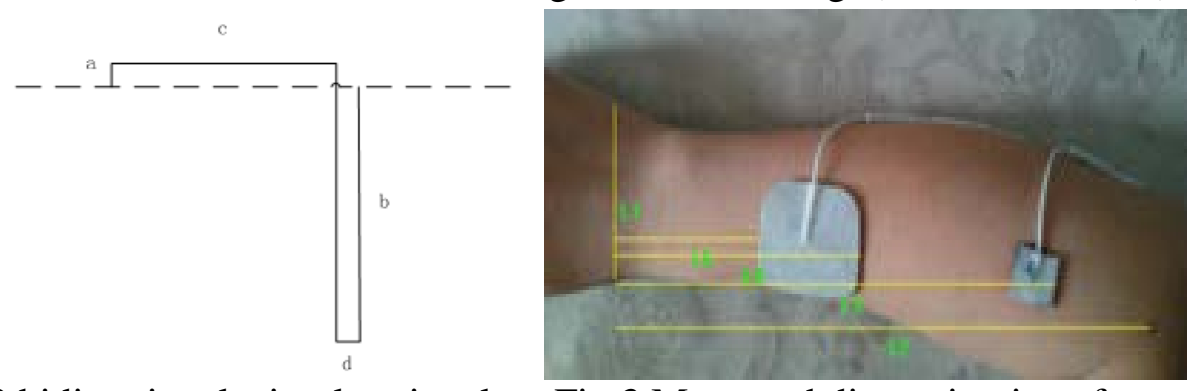

Fig 2 bidirectional stimulus signal Fig 3 Measured dimension in software

Experiment steps. Experimental instruments involved in this experiment were AMPI Master-9 programmable stimulator, AMPI ISO-Flex stimulus isolatorand self adhesive electrodes. Five subjects entered the experiment after resting. Larger electrode is a reference electrode, attached on the forearm near hand, the smaller electrode is stimulating electrode, attached to the forearm extensor carpi group.

Firstly, the stimulating electrode was moved from shou san li to shang lian point by point to look for the output of pure action of wrist action. Under the initial excitation signal, the amplitude of the current was fine tuned to observe whether there was a trend of wrist extension movement and the position of the electrode was fine tune to make the movement more harmonious and comfortable. At this point, the electrodes position was taken, and the Image-Pro software was introduced to measure the coordinates of the site. Then, we recorded the threshold current of wrist extension action under the fixed frequency and different width pulse. Secondly, two wrist extension action sites were found at proper pulse width in the forearm of each subject. The forearm length, the position of the stimulating electrode and the upper and lower edge sites of the reference electrode 
were recorded. The upper edge of reference electrode means near the cubital end. Finally, we summarized the data from five subjects on two sites respectively.

\section{Result}

Data aggregation for sites measurement is shown in Table 1. The mean position of excitating electrode of site 1 is located 9.76 inch to the wrist stripes, which is about 2 inches under the horizontal elbow. The mean position of reference electrode (upper) is 4.91 inch to wrist stripes and that of reference electrode (upper) is 2.75 inch to wrist stripes. According to the country standard location of acupoints, Shou san li(LI10) located in the back of the forearm radials, on the lines of Yangxi and Quchi and below the elbow stripes 2 inchs. Wen liu(LI7) and Pianli(LI6) located in the back of the forearm radials, on the lines of Yangxi and Quchi and up to wrist stripes 5 and 3 inches respectively. The comparison result showed that the position of the excitation electrode is shou san li(LI10) and that of reference electrode is between Wen liu(LI7) and Pianli(LI6).

Similar to the conclusions mentioned above, the mean position of excitating electrode of site 2 is located 8.89 inch to the wrist stripes, which is about 3 inches under the horizontal elbow. According to the country standard location of acupoints, Shang lian(LI9) located in the back of the forearm radials, on the lines of Yangxi and Quchi and below the elbow stripes 3 inchs. The comparison result showed that the position of the excitation electrode is Shang lian(LI9).

Table 1 data aggregation for site measurement

\begin{tabular}{|c|c|c|c|c|c|c|}
\hline \multirow{2}{*}{ subjects } & \multicolumn{2}{|c|}{ Exciting electrode (inch) } & \multirow{2}{*}{$\begin{array}{c}\begin{array}{r}\text { Reference } \\
\text { (upper) }\end{array} \\
\text { Site } 1\end{array}$} & \multirow{2}{*}{$\begin{array}{c}\begin{array}{c}\text { electrode } \\
\text { (inch) }\end{array} \\
\text { Site } 2\end{array}$} & \multicolumn{2}{|c|}{$\begin{array}{l}\text { Reference electrode } \\
\text { (lower) (inch) }\end{array}$} \\
\hline & Site 1 & Site 2 & & & Site 1 & Site 2 \\
\hline 1 & 10.00 & 9.29 & 4.80 & 4.38 & 2.40 & 4.38 \\
\hline 2 & 10.35 & 9.48 & 5.25 & 5.28 & 3.04 & 5.28 \\
\hline 3 & 9.84 & 9.05 & 5.46 & 5.59 & 3.21 & 5.59 \\
\hline 4 & 9.16 & 8.26 & 3.99 & 3.98 & 1.95 & 3.98 \\
\hline 5 & 9.44 & 8.39 & 5.06 & 5.17 & 3.16 & 5.17 \\
\hline$\overline{\mathrm{m}} \pm \mathrm{SD}$ & $9.76 \pm 0.47$ & $8.89 \pm 0.54$ & $4.91 \pm 0.57$ & $4.88 \pm 0.67$ & $2.75 \pm 0.55$ & $4.88 \pm 0.67$ \\
\hline
\end{tabular}

\section{Conclusion}

This site detection experiments proved the possibility and reliability of the pure action signal can be obtained by using the reasonable stimulation signal and the acupoint point of the muscle group. With strong guidance, the detection method is also applicable to other movements, such as wrist flexion, elbow extension and flexion. But the acupoints of the human body has a broad scope and is not a very precise loci. In order to achieve a better effect, the position of the excitation electrode should be fine tuned around the acupoints calculated by proportional bone measurement. The determination of pure action sites laid the foundation for the acquisition, coding and classification of different action EMG signals in the future.

\section{Acknowledgements}

This work was supported by Natural fund of Gansu Province B(2014qs03392).

\section{References}

[1] XU Jia , XIE Li. Research of the Surface Electromyography in the Hemiplegic ower Limbs of the Post-Stroke Patients[J], Chinese Journal of disability, 2012, 20(2):5-7. 
[2] YANG Tangzhu, HUANG Xiaolin, XIONG Caihua, et al. Surface electromyographic analysis during five kinds of activities of daily living in normal subjects and hemiplegic patients[J], Chinese Journal of Rehabilitation Medicine, 2011,26(3):236-239.

[3] HE Wen, WANG Kai. Advance in Rehabilitation of Upper Limb Function in Hemiplegic Patients after Stroke (review)[J], Chinese Journal of Rehabilitation Theory and Practice, 2014,20(4):334-339.

[4] Weingarden H, Ring H. Functional electrical stimulation induced neural changes and recovery after stroke[J]. Eura Medicophys,2006,42(2):87-90

[5] State Administration of China Standardization. Catalogue of national standards of the people's republic of China[M]. Beijing, China Quality Inspection press, 2010:305. 